\title{
Establishment of Novel Neuroendocrine Carcinoma Patient-Derived Xenograft Models for Receptor Peptide-Targeted Therapy
}

Catherine G. Tran ${ }^{1 \#}$, Luis C. Borbon ${ }^{1 \#}$, Jacqueline L. Mudd², Ellen Abusada ${ }^{3}$, Solmaz AghaAmiri ${ }^{4}$, Sukhen C. Ghosh ${ }^{4}$, Servando Hernandez Vargas ${ }^{4}$, Guiying Li ${ }^{1}$, Gabriella V. Beyer ${ }^{1}$, Mary McDonough ${ }^{1}$, Rachel Li ${ }^{1}$, Carlos H.F. Chan ${ }^{1}$, Susan A. Walsh ${ }^{5}$, Thaddeus J. Wadas ${ }^{5}$, Thomas O'Dorisio ${ }^{6}$, M. Sue O'Dorisio ${ }^{7}$, Ramaswamy Govindan ${ }^{8}$, Paul F. Cliften ${ }^{9}$, Ali Azhdarinia $^{4}$, Andrew M. Bellizzi ${ }^{3}$, Ryan C. Fields ${ }^{2}$, James R. Howe ${ }^{1^{*}}$, and Po Hien Ear ${ }^{1^{*}}$

1 Department of Surgery, University of lowa Carver College of Medicine, lowa City, IA 2 Department of Surgery, Washington University School of Medicine, St. Louis, Missouri 3 Department of Pathology, University of lowa Carver College of Medicine, lowa City, IA 4 The Brown Foundation Institute of Molecular Medicine, McGovern Medical School, The University of Texas Health Science Center at Houston, Houston, Texas.

5 Department of Radiology, University of lowa Carver College of Medicine, lowa City, IA 6 Department of Internal Medicine, University of lowa Carver College of Medicine, lowa City, IA 7 Department of Pediatrics, University of lowa Carver College of Medicine, lowa City, IA 8 Department of Medicine, Washington University School of Medicine, St. Louis, Missouri 9 Department of Genetics, Washington University School of Medicine, St. Louis, Missouri

\# These authors contributed equally to this manuscript.

* Correspondence should be addressed to James R. Howe or Po Hien Ear.

James R. Howe, MD

Professor of Surgery

Department of Surgery, Section of Surgical Oncology and Endocrine Surgery

University of lowa Carver College of Medicine

200 Hawkins Drive

lowa City, IA 52242

Phone : 319-356-1727

Fax : 319-353-8940

Email : james-howe@uiowa.edu

Po Hien Ear, PhD

Research Assistant Professor

Department of Surgery, Section of Surgical Oncology and Endocrine Surgery

University of lowa Carver College of Medicine

375 Newton Road, MERF 5206

lowa City, IA 52242

Phone: (319) 335-8983

Email: pohien-ear@uiowa.edu 


\section{Simple Summary}

Gastroenteropancreatic neuroendocrine neoplasms (GEP NENs) are a family of rare cancers with rising incidence in recent years. GEP NEN tumor cells are difficult to propagate and few cellular and patient-derived xenograft (PDX) models of GEP NENs are available for testing new therapies and studying the heterogeneous nature of these cancers. Here, we described the establishment and characterization of 2 novel NEC cellular and PDX models (NEC913 and NEC1452). NEC913 PDX tumors express somatostatin receptor 2 (SSTR2) whereas NEC1452 PDX tumors are SSTR2 negative. As a proof-of-concept study, we demonstrated how these PDX models can be used for peptide imaging experiments targeting SSTR2 using fluorescentlylabelled octreotide. The NEC913 and NEC1452 PDX lines represent valuable new tools for accelerating the process of drug discovery for GEP NENs. 


\begin{abstract}
Gastroenteropancreatic neuroendocrine neoplasms (GEP NENs) are rare cancers consisting of neuroendocrine carcinomas (NECs) and neuroendocrine tumors (NETs), which have been increasing in incidence in recent years. Few cell lines and pre-clinical models exist for the study of GEP NECs and NETs, limiting the ability to discover novel imaging and treatment modalities. To address this gap, we isolated tumor cells from cryopreserved patient GEP NECs and NETs and injected them into the flanks of immunocompromised mice to establish patient-derived xenograft (PDX) models. Two of 6 mice developed tumors (NEC913 and NEC1452). Over 90\% of NEC913 and NEC1452 tumor cells stained positive for Ki67. NEC913 PDX tumors expressed neuroendocrine markers such as chromogranin $A(\mathrm{CgA})$, synaptophysin (SYP), and somatostatin receptor-2 (SSTR2) whereas NEC1452 PDX tumors do not express SSTR2. Exome sequencing revealed loss of $\mathrm{p} 53$ and RB1 in both tumors. To demonstrate an application of these novel NEC PDX models for SSTR2-targeted peptide imaging, the NEC913 and NEC1452 cells were bilaterally injected into mice. Near infrared-labelled octreotide was administered and fluorescent signal was specifically observed for the NEC913 SSTR2 positive tumors. These 2 GEP NEC PDX models serve as valuable resource for GEP NEN therapy testing.
\end{abstract}




\section{Introduction}

Tumors can arise within neuroendocrine cells throughout the body, and some of the most common sites that lead to human morbidity and mortality originate within the GI system, most commonly within the small bowel and pancreas, collectively known as gastroenteropancreatic neuroendocrine neoplasms (GEP NENs). The age-adjusted incidence of GEP NENs has increased 6.4-fold in the United States between 1973 and 2012, with an annual incidence of 6.98 per 100,000 persons. ${ }^{1}$ These tumors are typically slowly growing, but over $60 \%$ of patients present with metastatic disease, where survival is greatly diminished..$^{2-4}$ In addition to the stage of the disease, both tumor grade and cell morphology/degree of differentiation are also powerful predictors of survival. Based on the 2019 World Health Organization classification of neuroendocrine neoplasms, GEP NEN are comprised of well-differentiated neuroendocrine tumors (NETs) and poorly-differentiated neuroendocrine carcinomas (NECs). ${ }^{5}$ NECs are high grade tumors characterized by a Ki-67 proliferation index $>20 \%$ and a mitotic rate of over 20 mitoses per $2 \mathrm{~mm}^{2}$, although NEC tumors are frequently found to have Ki-67 indices $>50 \%{ }^{5}$ In addition to these distinguishing features of poor differentiation and high Ki-67, GEP NECs have frequent mutations in TP53 and RB1, while mutations in NET tumors are less common, and include MEN1, DAXX, and ATRX for pancreatic NETs (PNETs) and CDKN1B and 18q loss in small bowel NETS (SBNETs). ${ }^{6-9}$

Clinically, NECs are very aggressive, rapidly dividing tumors. They are associated with high rates of metastatic disease at presentation and poor prognosis. The incidence of NECs is not as well-defined due to changes in the WHO classification over the past decade, but epidemiological studies estimate this to be about $0.4 / 100,000$ person-years. ${ }^{10,11}$ The optimal treatment for GEP NECs has not been established, due in part to the rarity of these tumors and difficulty with performing randomized trials. As a result, current guidelines for treatment of GEP NEC are currently based on lower-level evidence (NCCN Guidelines 2021). ${ }^{12}$ Therapeutic 
strategies are largely derived from experience in management of small cell lung cancer, due to the pathologic and immunohistochemical similarities between small cell lung cancer and GEP NECs. ${ }^{13-15}$ The appropriateness of this has been called into question, as small cell lung cancer differs from NECs in several ways, including higher association with smoking, higher rate of brain metastases, and higher sensitivity to platinum-based chemotherapy. ${ }^{14-17}$ Patients are generally treated with chemotherapy regimens including platinum-based alkylating agents (carboplatin) and topoisomerase inhibitors (etoposide). Despite treatment, response rates are only $30-50 \%$ and median overall survival is 9 to 20 months ${ }^{16-18}$.

Current therapies for both NETs and NECs are limited to somatostatin analogues (SSAs), mTOR inhibitors (everolimus), tyrosine kinase inhibitors (sunitinib), limited chemotherapy regimens, and radiolabeled SSAs. ${ }^{19}$ One of the biggest barriers to identifying additional active therapeutics has been the inability to establish GEP NEN cell lines that can be grown robustly. Some of these tumor cells can be grown in culture as spheroids, but they grow very slowly and are difficult to propagate as xenografts. ${ }^{20-22}$ The 2 widely used cell lines, BON ${ }^{23}$ and $\mathrm{QGP} 1^{24}$ resemble poorly differentiated $\mathrm{NECs}^{25-27}$, and unfortunately express low level of NEN markers such as the somatostatin receptor 2 (SSTR2). ${ }^{25}$ Although well-differentiated GEP NET cell lines such as the P-STS, GOT1, and NT-3 cells have been described ${ }^{25,26,28}$, difficulties growing these cells in abundance have limited their distribution to other researchers. The paucity of available cell lines has been a significant hurdle towards better understanding NEN biology and to provide theragnostic models, and therefore we set out to establish new cell lines to expand these options for NEN research. 


\section{Methods}

Cell lines and patient-derived xenograft models

The inventory of the Washington University PDX center was searched for neuroendocrine tumors and carcinomas, which were collected under an Institutional Review Board-approved protocol (\#201708051) of Washington University and cryo-preserved. Six patient samples were found with suitable amounts of frozen tissue for further study. Tumors were thawed, minced, digested with collagenase, and strained to obtain a single-cell suspension. One to ten thousand cells were injected subcutaneously into NOD scid gamma (NSG) mice under an Institutional Animal Care and Use Committee-approved protocol (\#9051771). Once visible subcutaneous tumors developed, tumors were harvested, processed, and injected into another generation of NSG mice and placed in single cell suspension culture. Tumor cells were grown in Dulbecco's modified Eagle's medium (DMEM)/F12 with 10\% fetal bovine serum (FBS), $1 \%$ penicillin/streptomycin, $1 \% \mathrm{~L}$-glutamine, insulin, and nicotinamide. ${ }^{20} \mathrm{BON}$ cells were cultured in Dulbecco's modified Eagle's medium (DMEM)/F12 with 10\% fetal bovine serum (FBS), $1 \%$ penicillin/streptomycin, and $1 \%$ L-glutamine. ${ }^{23}$

\section{Histology and immunohistochemistry}

Patient samples from surgery were fixed in formalin, embedded in paraffin, and sectioned. Slides were deparaffinized, rehydrated, and stained with hematoxylin and eosin (H\&E). Slides were immunostained using specific antibody against chromogranin $\mathrm{A}(\mathrm{CgA})$ (Thermo Scientific, \#MA5-13096), synaptophysin (SYP) (Agilent Dako, \#M7315), achaete-scute family bHLH transcription factor 1 (ASCL1) (BD Pharmingen, \#556604), p53 (Agilent Dako, \#M700101-2), retinoblastoma protein $(\mathrm{Rb})$ (BD Pharmingen, \#554136), somatostatin receptor 2 (SSTR2) (Abcam, \#ab134152), C-X-C motif chemokine receptor 4 (CXCR4) (Abcam, \#ab124824), and Ki-67 (Agilent Dako, \#M724001-2). Ki-67 proliferation index was quantified by percentage of positively staining cells in $\sim 500$ tumor cells per tumor sample. 


\section{Quantitative PCR}

RNA was extracted from tumors grown in animals using the RNeasy Plus Universal Kit (Qiagen) and reverse transcribed to cDNA using qScript cDNA Supermix (QuantaBio). Quantitative PCR was performed with gene-specific primers and PerfeCTa SYBR Green Supermix dye (Quantabio) using the 7900HT Fast Real-Time PCR System (Applied Biosystems). Primer sequences were obtained from PrimerBank (https://pga.mgh.harvard.edu/primerbank/) and purchased from Integrated DNA Technologies (IDT). Primer sequences used for qPCR analysis are:

$\begin{array}{lll}\text { Gene symbol } & \text { Forward } & \text { Reverse } \\ \text { GAPDH } & \text { GGAGCGAGATCCCTCCAAAT } & \text { GGCTGTTGTCATACTTCTCATGG } \\ \text { CGA } & \text { TAAAGGGGATACCGAGGTGATG } & \text { TCGGAGTGTCTCAAAACATTCC } \\ \text { SYP } & \text { CTCGGCTTTGTGAAGGTCT } & \text { CTGAGGTCACTCTCGGTCTTG } \\ \text { SSTR1 } & \text { GCGCCATCCTGATCTCTTTCA } & \text { AACGTGGAGGTGACTAGGAAG } \\ \text { SSTR2 } & \text { TGGCTATCCATTCCATTTGACC } & \text { AGGACTGCATTGCTTGTCAGG } \\ \text { SSTR3 } & \text { AGAACCTGAGAATGCCTCCTC } & \text { GCCGCAGGACCACATAGATG } \\ \text { SSTR4 } & \text { GCATGGTCGCTATCCAGTG } & \text { GCGAAGGATCACGAAGATGAC } \\ \text { SSTR5 } & \text { GTGATCCTTCGCTACGCCAA } & \text { CACGGTGAGACAGAAGACGC } \\ \text { CXCR4 } & \text { ACGCCACCAACAGTCAGAG } & \text { AGTCGGGAATAGTCAGCAGGA }\end{array}$

Gene expression levels were normalized to the control gene GAPDH to determine the relative fold change. Statistical analyses of gene expression changes were performed using T-tests in Prism GraphPad. P $<0.05$ was depicted with *. $\mathrm{P}<0.01$ was depicted with **.

\section{Immunofluorescence}

Cells derived from animal tumors were fixed with $4 \%$ paraformaldehyde for 10 minutes, then stained with primary antibody SYP (Abcam, \#32127) at 1:600 dilution, CgA (Invitrogen, \#MA513096) at 1:400 dilution, and SSTR2 (Sigma, \#HPA007264) at 1:400 dilution overnight. Cells were washed and incubated with a FITC-conjugated secondary antibodies (Jackson 
ImmunoResearch, \#115-095-062 and \#711-095-152) at 1:500 dilution for 1 hour. Immunofluorescent images were taken using a fluorescent microscope at $200 \mathrm{~ms}$ exposure time.

\section{Genomic DNA analyses}

Genomic DNA from PDX tumors, and peripheral blood mononuclear cells were extracted using a DNA extraction kit (Qiagen). Short tandem repeats analyses were performed on the DNA samples using the Cell Check9 panel of 9 human simple tandem repeat polymorphisms (IDEXX). Exome sequencings of PDX tumors were performed by submitting genomic DNA from NEC913 and NEC1452 PDX tumors to the Washington University Genome Technology Access Center for analyses with the IDT Exome 150X coverage. Exome sequencing data were analyzed using a DRAGEN processor and compared to the GRCh38 reference genome.

\section{Imaging of patient-derived xenograft mouse model with bilateral tumors}

Five female NSG mice (Jackson Laboratory, Stock no: 005557) were anesthetized with 1\% to $2 \%$ isoflurane at 10 weeks of age, and were subcutaneously injected with $1 \times 10^{6}$ SSTR2(+) cells in Matrigel (Corning, \#356235) in the left shoulder, and $1 \times 10^{6}$ SSTR2(-) cells in the right shoulder. When bilateral tumor size reached between 10 to $20 \mathrm{~mm}$ in diameter at 5 weeks postimplantation, in vivo and ex vivo NIR fluorescence imaging were conducted with NIR-TOC as described by Hernandez-Vargas et al. ${ }^{29,30}$ In brief, 6 nmol of NIR-TOC diluted in $100 \mu$ L PBS was administered via mouse tail-vein injection 24 hours prior to imaging studies. NIR fluorescence imaging was acquired using the IVIS Lumina S5 small animal imaging station and Living Image ${ }^{\circledR}$ software (PerkinElmer, Waltham, MA, USA) with excitation and emission set to 740 and $790 \mathrm{~nm}$, respectively. Images with favorable contrast-to-noise ratio were obtained using exposure time of 2 seconds for in-vivo and 0.1 seconds for ex-vivo imaging, with subject height 
of $1.50 \mathrm{~cm}$, small binning and F/Stop setting of 2 , and field of view setting $\mathrm{C}$. After completing in vivo imaging, mice were euthanized and dissection was immediately performed for ex vivo isolation and imaging of subcutaneous tumors as well as major intraabdominal and intrathoracic organs. Quantification of NIR fluorescent signal was performed using ImageJ version 1.53a (NIH, MD, USA). Statistical analyses for NIR fluorescent signal were performed using T-tests in Prism GraphPad. P $<0.05$ was depicted with *. 


\section{Results}

Tumor cells from 6 cryopreserved patient tumors (Table 1) were injected into the flank of NSG mice to generate patient-derived xenograft (PDX) models (Figure 1A).

Table 1. List of GEP NEN patient tumor samples used for patientderived xenograft (PDX) development

\begin{tabular}{|l|c|c|c|}
\hline $\begin{array}{l}\text { Patient Tumor ID } \\
\text { Number }\end{array}$ & $\begin{array}{c}\text { Classification of } \\
\text { tumor }\end{array}$ & $\begin{array}{c}\text { Establishment } \\
\text { of PDX }\end{array}$ & Tumor Grade \\
\hline PNET459 & Pancreatic NET & no & G2 \\
\hline PNET560 & Pancreatic NET & no & G2 \\
\hline PNET1164 & Pancreatic NET & no & G2 \\
\hline SBNET1063 & Small bowel NET & no & G3 \\
\hline NEC913 & Ampullary NEC & yes & G3 \\
\hline NEC1452 & Retroperitoneal NEC & yes & G3 \\
\hline
\end{tabular}

At 3-months post tumor cell injection, 2 mice harboring GEP NEC cells had developed subcutaneous tumors of approximately $1 \mathrm{~cm}$ in diameter (NEC913 and NEC1452; Figure 1A) while 4 GEP NET patient tumor cell samples injected into mice did not form tumor (Table 1). Subcutaneous injection of $1 \times 10^{6}$ NEC913 and NEC1452 cells grew into tumors about 1000 $\mathrm{mm}^{3}$ and $1500 \mathrm{~mm}^{3}$ in size, respectively, after 5 weeks in subsequent passages. The tumor formation rate is $100 \%$. The NEC913 and NEC1452 xenograft tumors were harvested and collected for histological and biochemical analyses. A separate portion of the NEC913 and NEC1452 tumors were collected for tumor cell isolation and injection into another generation of mice for propagation of the PDX models and for establishment of cell lines. Both NEC913 and NEC1452 cells could be successfully maintained in culture for months in enriched DMEM/F12 medium. Both PDX tumors stained positive for the neuroendocrine tumor markers chromogranin A ( $\mathrm{CgA}$ ) and synaptophysin (SYP), but only the NEC913 PDX tumor stained positive for somatostatin receptor 2 (SSTR2; Figure 1B). Exome sequencing of the NEC913 and NEC1452 PDX tumors were performed and mutations in TP53 and RB1 were confirmed (Figure 1C; Supplementary Tables 1 \& 2). 


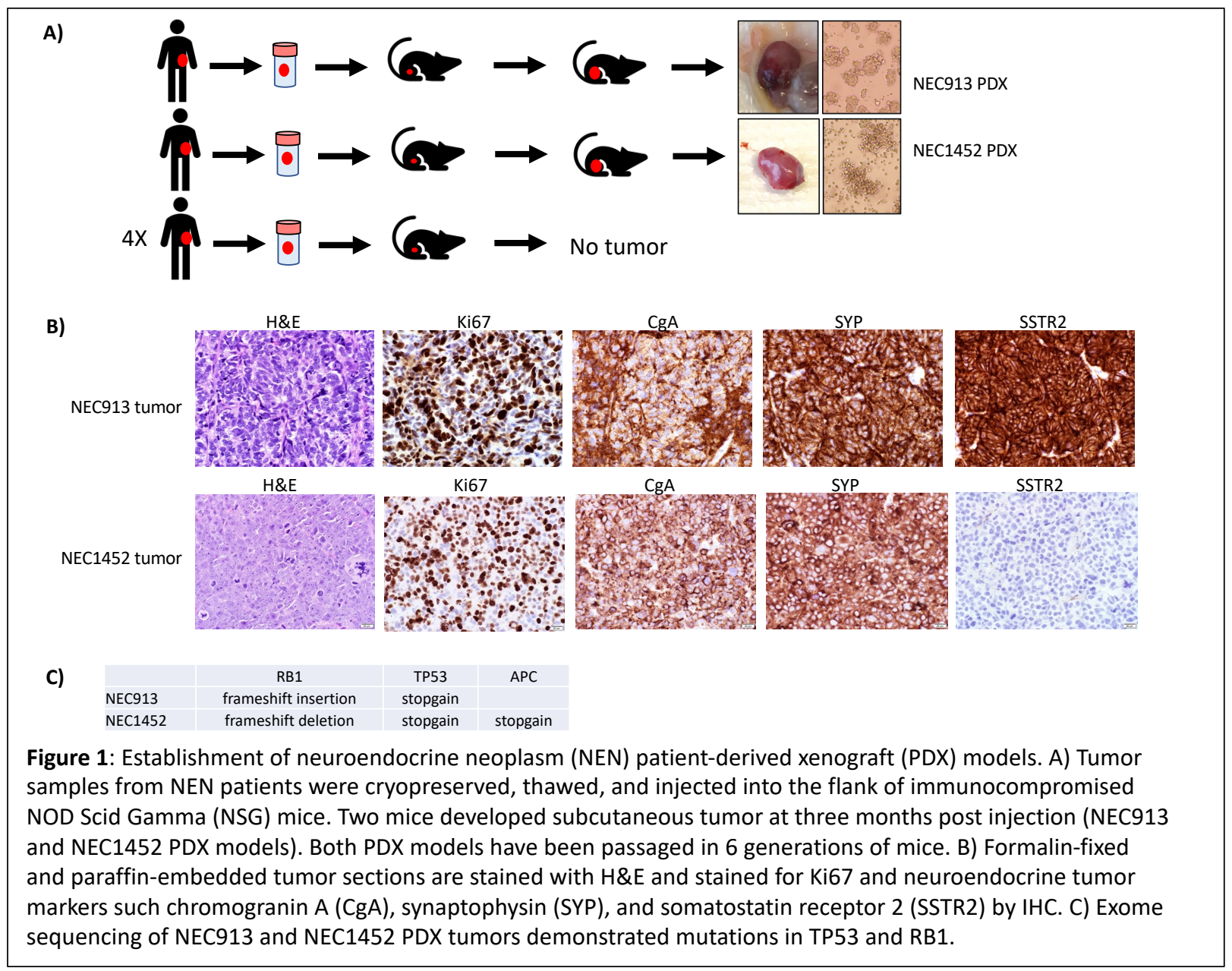

We were able to retrieve the original patient tumor for the NEC913 line for comparison with the PDX model. The NEC913 tumor came from a patient presenting with jaundice and upper GI bleeding. A biopsy revealed a G3 NEC of the ampulla and the patient was treated with carboplatin/etoposide chemotherapy for 4 months, then had a Whipple procedure, where a 2.5 $\mathrm{cm}$ primary tumor with multiple involved nodes were also removed (Figure 2A). Hematoxylin and eosin (H\&E) staining showed the presence of both small and large NEC cells (Figure 2B). The NEC913 primary tumor stained positive for Ki-67 in 90\% of cells (Figure 2C). Outside pathology was TTF-1 positive and CDX-2 negative; additional staining here was positive for CgA, SYP, SSTR2, and ASCL1 (Figure 2D-G). A low level of p53 was detected (Figure 2H) however exome sequencing data identified several stopgain mutations where the first stopgain mutation is located in codon 147 of TP53 (Figure 1C \& Supplementary Table 1) suggesting 
that the IHC staining detects only the first 146 amino acid fragment of p53. Expression of Rb was lost (Figure 2l).
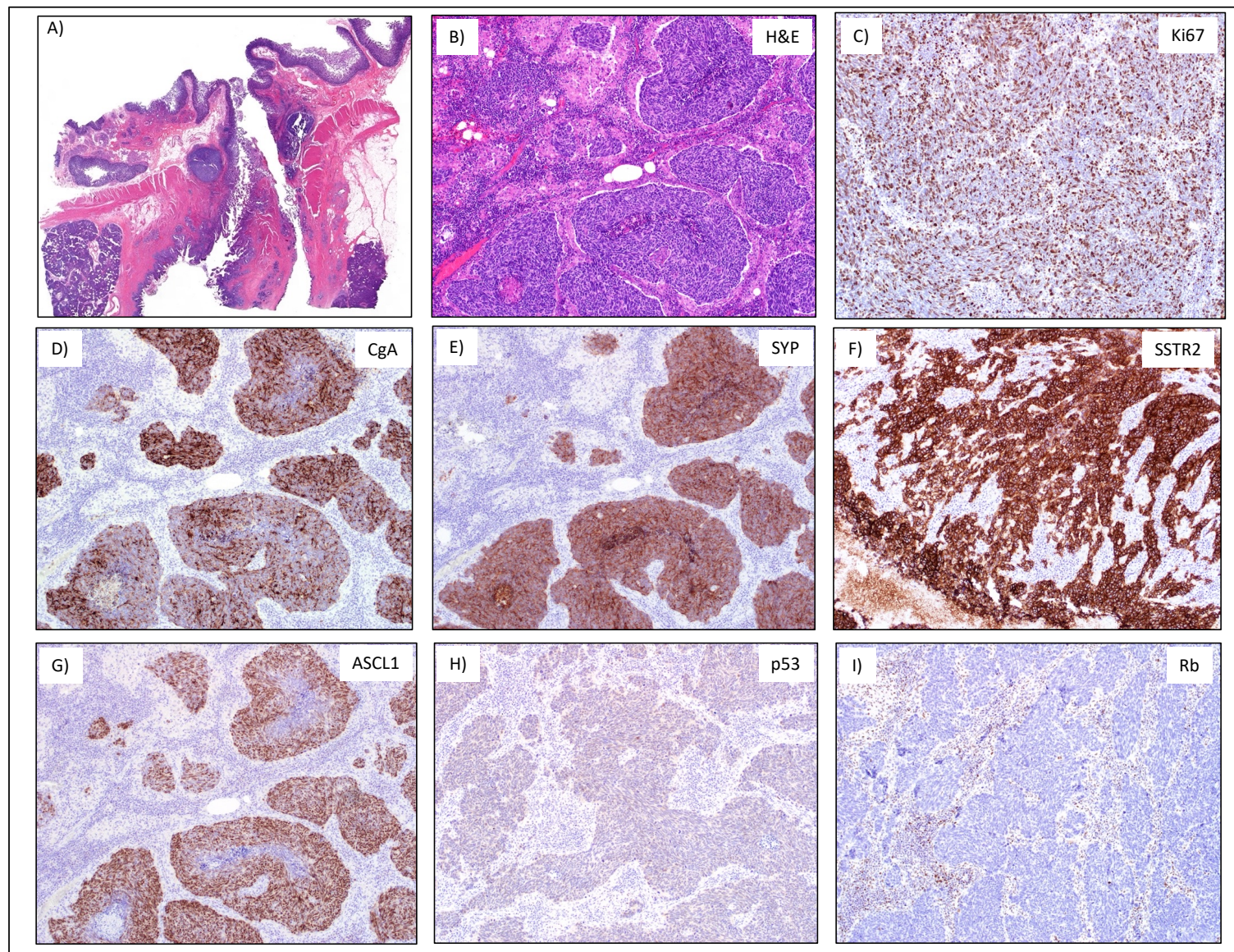

Figure 2: IHC analyses of NEC913 patient sample. A) Primary NEC tumor at the ampulla of Vater. B) H\&E staining of primary NEC tumor. C-I) Staining for Ki67, CgA, SYP, SSTR2, ASCL1, p53, and RB.

Exome sequencing of the NEC913 PDX tumor revealed an RB frameshift insertion (1091_1092insCG) leading to a premature stop codon (Figure 1C \& Supplementary Table 1).

Short tandem repeat analyses confirmed that the NEC913 patient blood sample shared the same alleles with the NEC913 PDX tumor (Supplementary Table 3) and that these samples did not match any of the existing research samples in the IDEXX DSMZ STR database meaning that these patient samples are being reported for the first time.

The patient giving rise to tumor NEC1452 presented with a mediastinal and supraclavicular masses, as well as liver, pancreatic, retroperitoneal, and rectal lesions that were FDG-PET 
positive and only mildly DOTATATE PET avid. Supraclavicular node biopsy showed small cell G3 NEC and carboplatin/etoposide were started, followed by FOLFIRI after progression, then immunotherapy. A retroperitoneal node was biopsied due to poor response, which showed large cell NEC with a Ki-67 of $80-90 \%$, which was the source of this PDX. The treating Medical Oncologist considered the rectum to be the primary site, because this had the highest FDG-PET avidity, with uptake in perirectal nodes, a nearly obstructing mass seen on sigmoidoscopy, and the presence of $A P C$ mutations in the tumor (Figure 1C \& Supplementary Figure 2). The NEC1452 PDX tumor sample did not match any pre-existing samples in the IDEXX DSMZ STR database (Supplementary Table 4), and exome sequencing confirmed TP53 stopgain mutation and RB frameshift mutation (Figure 1C \& Supplementary Table 2).

A)

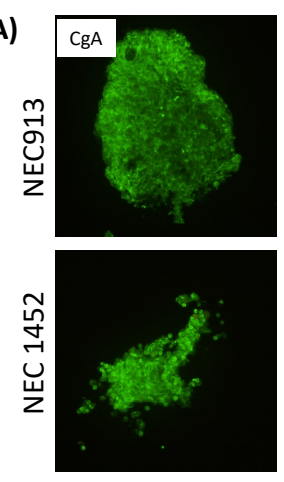

D)

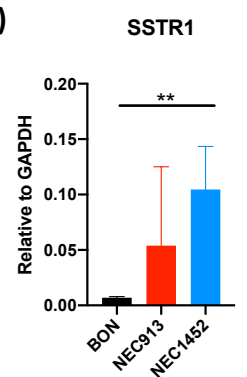

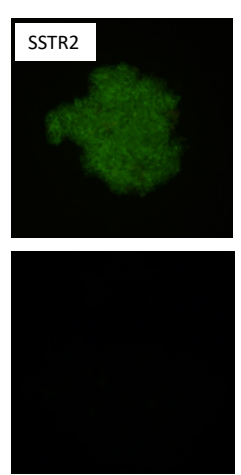

E)

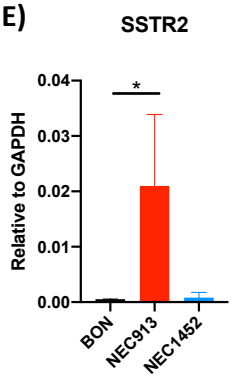

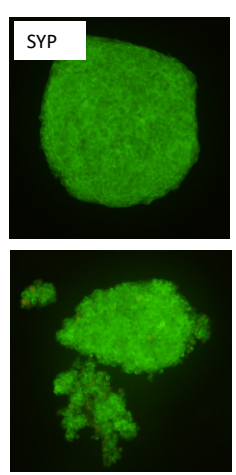

F)

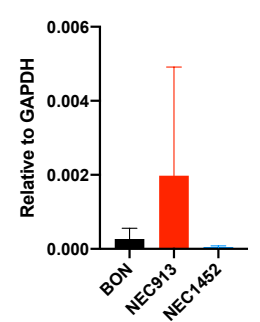

B)

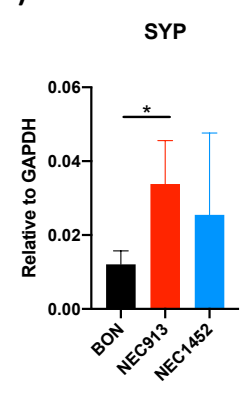

G)

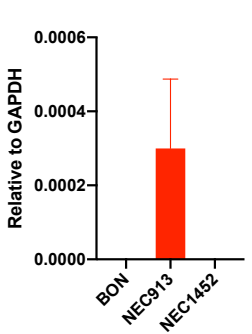

C)

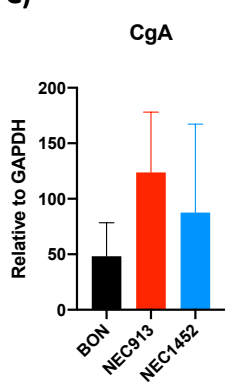

H) SSTR5

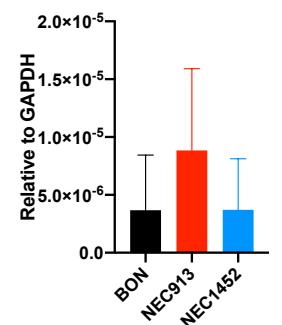

Figure 3: Characterization of NEC913 and NEC1452 cells for NET markers. A) NEC913 and NEC1452 cells are stained with antibodies against CgA (1/300), SSTR2 (1/300), and SYP (1/600) overnight and with secondary antibodies coupled to FITC (1/500) for $1 \mathrm{hr}$ at room temperature. Microscopy pictures are taken using $200 \mathrm{~ms}$ exposure time. B-H) Gene expression analyses of NET markers in NEC913 and NEC1452 cells compared to BON cells.

NEC913 and NEC1452 tumor cells grow robustly as suspension cultures or as spheroids embedded in extracellular matrix. By immunofluorescent staining, we confirmed the expression of CgA and SYP in both cell lines and SSTR2 in only the NEC913 line (Figure 3A). To further 
characterize these novel cell lines for additional neuroendocrine cancer markers, gene expression analyses using quantitative PCR was performed (Figure 3B-H). In comparison to the established BON cells, NEC913 was found to have significantly increased SYP and SSTR2 expression (Figure 3B\&E). NEC1452 cells were determined to have increased SSTR1 expression relative to BON cells (Figure 3D).

To demonstrate the utility of NEC PDX models as a potential tool for testing receptor-targeted theranostics, we conducted a proof-of concept study whereby we established a mouse model with tumor implantations using the NEC913 (SSTR2+) and NEC1452 (SSTR2-) cells in opposite shoulders for SSTR2-targeted imaging (Figure 4A).

In vivo NIR Imaging Overlay
Figure 4: Application of NEC PDX models for SSTR2-targeted imaging. A) Representative photograph of mice
harboring NEC913 and NEC1452 tumors ranging from 1.0 to $2.0 \mathrm{~cm}$ in diameter 5 weeks post tumor cell
injections from an $\mathrm{n}=5$ mice experiment. B) Near infrared (NIR) fluorescence imaging of mice harboring
NEC913 and NEC1452 tumors using exposure time of 2 seconds and excitation and emission wavelengths set at
740 and $790 \mathrm{~nm}$, respectively. NEC913 tumors are circled in red and NEC1452 tumors are circled in blue. C)
Representative ex vivo NIR fluorescence imaging of dissected NEC913 and NEC1452 tumors from an $\mathrm{n}=5$ mice
experiment. D-E) Quantifications of in vivo and ex vivo NIR signal in NEC913 and NEC1452 tumors.

We then injected these mice with a near-infrared octreotide analog (NIR-TOC) which previously demonstrated to specifically detects SSTR2 on NEN cells, and imaged them using near infrared (NIR) fluorescence imaging. ${ }^{29,30}$ Image analysis revealed that NIR-fluorescence signal was 
localized only in the NEC913(SSTR2+) tumor (Figure 4B). To confirm the localization of NIR fluorescence signal on the NEC tumors, ex-vivo NIR fluorescence imaging was performed on both tumors after they were removed from the animals. The NIR fluorescence signal was detected in the NEC913 tumor but not NEC1452 (Figure 4C) corroborating the in vivo imaging results (Figure 4B). Qualitative assessment of SSTR2-mediated uptake was supported by semi-quantitative image analyses which revealed an approximately 2 -fold increase and a 3-fold increase in fluorescent signal intensity of NEC913 compared to NEC1452 tumors in the in vivo and ex-vivo experiments, respectively (Figure 4D\&E).

Further characterization of the NEC913 PDX tumor confirmed low expression of the truncated p53, and no expression of RB (Figure 5A), which is similar to the expression pattern observed in original patient tumor IHC analyses (Figure $\mathbf{2 H \& I}$ ). The expression of ASCL1 was lower in the NEC913 PDX tumor (Figure 5A) compared to the original tumor (Figure 2G). In addition, the NEC913 PDX tumor expressed CXCR4 (Figure 5A). The CXCR4 expression was also detected in NEC913 spheroids by quantitative PCR and IHC using specific antibody against CXCR4 (Figure 5B-C).
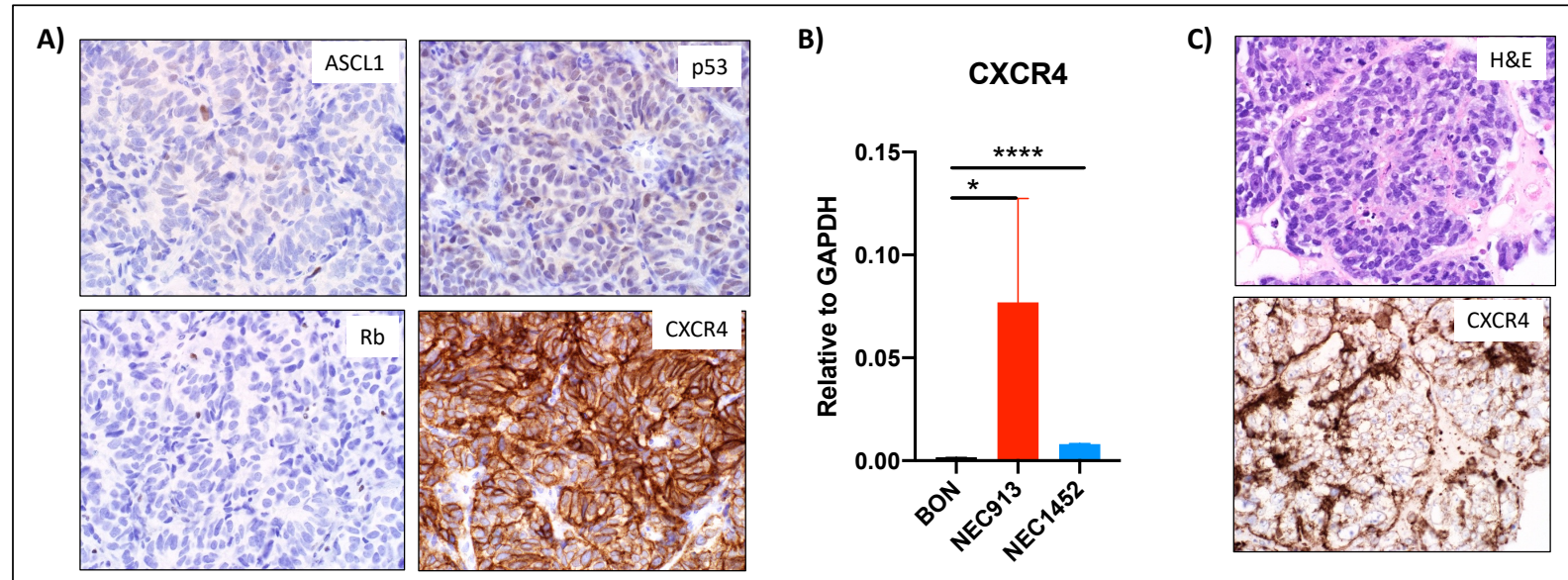

Figure 5: Additional characterization of NEC913 PDX model. A) IHC analyses of NEC913 PDX tumors for NEC markers such as Rb, p53, ASCL1, and CXCR4. B) Comparison of the gene expression levels of CXCR4 in BON and NEC913 cells by quantitative PCR. C) NEC913 spheroids H\&E staining and IHC analysis of CXCR4. 


\section{Discussion}

GEP NETs and NECs are rare cancers with few in vitro and in vivo models available for therapeutic testing. ${ }^{31}$ GEP NET cell lines and spheroids are difficult to propagate as they take approximately 2 to 3 weeks to divide..$^{20,23,25,32}$ A limited number of GEP NEC cell and organoid lines have been recently published but distribution remains limited. The 2 currently available human GEP-NEN-derived cell lines, BON and QGP-1, divide approximately every 3 days and carry TP53 and Rb mutations. ${ }^{26}$ They are morphologically poorly differentiated, and have Ki-67 rates that exceed $70-90 \%$, which define them as neuroendocrine carcinoma cell lines.

Because well-differentiated NETs grow slowly, attempts to propagate them long term have not generally been successful. We have shown that these can be grown in culture for up to 9 months and still remain well-differentiated and express NET markers such as synaptophysin, chromogranin and SSTR2. ${ }^{20}$ However, after about 2 weeks, growth remains fairly constant at a low level. In this study, we were unsuccessful in establishing all 4 frozen NET samples in culture or immunocompromised mice at 3 months post injection (Table 1). Considering that a majority of GEP NETs are generally slow-growing Grade 1 and Grade 2 tumors with a Ki-67 index less than $20 \%$, it is possible that a period longer than 3 months is required for tumor formation. Interestingly, even the high-grade SBNET sample SBNET1063 (Table 1), which was a WHO Grade 3 tumor, did not generate a subcutaneous tumor, suggesting that a xenograft model may not be ideal for NET PDX development. There have been a few SBNET cell lines described such as P-STS, GOT1, NT3. ${ }^{25,26,28}$ A PNET line has also been described Chamberlain et al. ${ }^{33}$ Although these appear be promising new GEP NET models, they have not been widely distributed to many investigators. The most likely explanation for this is that they cannot be grown in large enough numbers to share, or that over time the cells that do survive could potentially dedifferentiate into NECs. 
We were able to establish 2 new NEC cell lines which grow well in culture and could be grown through several generations in immunocompromised mice. These lines significantly expand the options for study of NECs, and are derived from different sites. The NEC913 is derived from an ampullary NEC and NEC1452 from a retroperitoneal node from a patient suspected to have a rectal NEC. Both NEC913 and NEC1452 PDX tumors contain TP53 and RB mutations which are commonly reported in GEP NEC..$^{8,34,35}$ Both lines expressed synaptophysin, chromogranin A, and had Ki-67 of $>90 \%$. The NEC913 line expressed SSTR2 and CXCR4 while NEC1452 did not. Tumors from these PDX models can robustly be passaged in more than 6 generations of mice with a $100 \%$ rate of tumor formation. NEC cells from both PDX models can be maintained in culture in media supplemented with insulin and nicotinamide as suspension cultures or spheroids embedded in extracellular matrix.

Several lung NEC lines ${ }^{36,37}$ and colon NEC lines such as the HROC47, SS-2, NEC-DUE1 \& 2 ${ }^{36,38-40}$ have been established but few pancreas and small bowel NEC have been reported. ${ }^{39}$ Considering the rarity of GEP NEC PDX and cell models, the NEC913 and NEC1452 PDX models developed in this study could be tremendously valuable for a variety of pre-clinical experiments. Here, we showed that the NEC913 line can be useful in confirming the target specificity of NIR-TOC with fluorescence imaging in a clinically relevant model, suggesting high translational potential (Figure 4). Since the NEC913 PDX model maintains high SSTR2 (Figure 1B) and CXCR4 (Figure 5A-B) expression after six generations of passages. The NEC913 cells grow as spheroids in suspension culture or embedded in extracellular matrix and recapitulate characteristics of the PDX tumor and stained positive for CXCR4 (Figure 5C). This cell line also shows great promise as a potential tool for future investigations involving peptide radionucleotide therapy (PRRT), including testing of combination therapies or highly potent alpha-emitter PRRT. ${ }^{41}$ CXCR4 is also emerging as a valuable target in atypical lung carcinoids, and can be targeted with the radiolabeled ligand Pentixafor. ${ }^{42,43}$ Thus, NEC913 could also 
serve as an effective pre-clinical model for PRRT directed at CXCR4. The development and characterization of these NEC913 and NEC452 PDX lines represent valuable new tools that could overcome the significant limitations of existing preclinical models in NEN research and accelerate the process of drug discovery. 


\section{Conclusion:}

Two novel NEC PDX models (NEC913 and NEC1452) were established from cryopreserved patient tumors. Tumors from these PDX models can robustly be passaged in immunocompromised mice. The NEC913 PDX model maintained SSTR2 expression after 6 generations of passages and can be visualized using NIR-TOC peptide. In addition, the NEC913 PDX model expresses high level of CXC4, which makes it potentially useful for CXCR4-targeted theranostics. NEC cells from both PDX models can be maintained in culture in media supplemented with insulin and nicotinamide. Considering the rarity of GEP NEC PDX and cell lines, the NEC913 and NEC1452 PDX models are valuable pre-clinical models for peptide imaging, drug testing experiments, and studying GEP NEC tumor biology. 


\section{Supplementary Materials:}

\section{Supplementary Table 1: List of curated mutations identified in NEC913 PDX tumors by exome}

\section{sequencing.}

\begin{tabular}{|c|c|c|c|c|c|c|c|c|c|}
\hline Chr & Start & End & Ref & Alt & avsnp150 & Func & Gene & ExonicFunc & AAChange \\
\hline chr2 & 212652834 & 212652834 & c & $\mathrm{T}$ & rs375361752 & exonic & ERBB4 & nonsynonymous SNV & 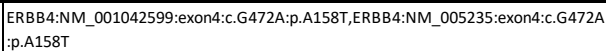 \\
\hline chr6 & 32188296 & 32188296 & c & $T$ & \begin{tabular}{|r|r|} 
rs561687277 \\
\end{tabular} & exonic & NOTCH4 & nonsynonymous SNV & NOTCH4:NM_004557:exon6:c.G1045A:p.G349S \\
\hline chr6 & 161533750 & 161533750 & c & G & nan & exonic & МАР3К4 & nonsynonymous SNV & $\begin{array}{l}\text { MAP3K4:NM_001363582:exon24:c.C4408G:p.R1470G,MAP3K4:NM_006724:exon24: } \\
\text { c.C4420G:p.R1474G,MAP3K4:NM_001301072:exon25:c.C4558G:p.R1520G,MAP3K4: } \\
\text { NM_005922:exon25:c.C4570G:p.R1524G,MAP3K4:NM_001291958:exon26:c.C2929 } \\
\text { G:p.R977G }\end{array}$ \\
\hline chr6 & 80717709 & 80717709 & G & c & nan & exonic & TTK & nonsynonymous SNV & $\begin{array}{l}\text { TTK:NM_001166691:exon3:c.G323C:p.S108T,TTK:NM_003318:exon3:c.G323C:p.S10 } \\
\text { 8T }\end{array}$ \\
\hline chr10 & 6527143 & 6527143 & $\mathrm{G}$ & A & rs2236379 & exonic & PRKCQ & nonsynonymous SNV & $\begin{array}{l}\text { PRKCQ:NM_001282645:exon9:c.C614T:p.P205L,PRKCQ:NM_001323266:exon9:C.C61 } \\
\text { 4T:p.P205LL,PRKCQ:NM_001242413:exon10:c.C989T:p.P330L,PRKCQ:NM_001282644 } \\
\text { :exon10:c.C881T:p.P294LLPRKCQ:NM_001323265:exon10:c.C989T:p.P330L,PRKCQ:N } \\
\text { M_001323267:exon10:c.C881T:p.P294L,PRKCQ:NM_006257:exon10:c.C989T:p.P330 } \\
\text { L }\end{array}$ \\
\hline chr11 & 108168057 & 108168057 & G & $\mathrm{T}$ & nan & exonic & ATM & nonsynonymous SNV & $\begin{array}{l}\text { ATM:NM_000051:exon33:c.G4953T:p.L1651F,ATM:NM_001351834:exon34:c.G4953 } \\
\text { T:p.L1651F }\end{array}$ \\
\hline chr11 & 62446348 & 62446348 & G & - & nan & exonic & UBXN1 & frameshift deletion & $\begin{array}{l}\text { UBXN1:NM_001286077:exon1:c.48delC:p.R17Gfs*23,UBXN1:NM_001286078:exon } \\
\text { 1:c.48deIC:p.R17Gfs*83,UBXN1:NM_015853:exon1:c.48delC:p.R17Gfs*23 }\end{array}$ \\
\hline \begin{tabular}{|c|} 
chr13 \\
\end{tabular} & 48942704 & 48942704 & - & CG & nan & exonic & RB1 & frameshift insertion & RB1:NM_000321:exon11:c.1091_1092insCG:p.E364Dfs*4 \\
\hline chr13 & 48942705 & 48942705 & - & GGT & nan & exonic & RB1 & nonframeshift insertion & RB1:NM_000321:exon11:c.1092_1093insGGT:p.E364_E365insG \\
\hline chr13 & 114782808 & 114782808 & G & A & rs781295846 & konic & RASA3 & stopgain & $\begin{array}{l}\text { RASA3:NM_001320822:exon12:c.C1015T:p.R339X,RASA3:NM_007368:exon12:c.C11 } \\
\text { 11T:p.R371X }\end{array}$ \\
\hline chr17 & 7577022 & 7577022 & G & A & rs121913344 & exonic & TP53 & stopgain & $\begin{array}{l}\text { TP53:NM_001126115:exon4:c.CC520T:p.R174X,TP53:NM_001126116:exon4:c.CS20T: } \\
\text { p.R174X,TP53:NM_001126117:exon4:C.C520T:p.R174X,TP53:NM_001276697:exon4: } \\
\text { c.C439T:p.R147X,TP53:NM_001276698:exon4:c.C439T:p.R147X,TP53:NM_00127669 } \\
\text { 9:exon4:c.C439T:p.R147X,_TP53:NM_001126118:exon7:c.C799T:p.R267X,TP53:NM_0 } \\
\text { 00546:exon8:c.C916T:p.R306X,TP53:NM_001126112:exon8:c.C916T:p.R306X,TP53:N } \\
\text { M_001126113:exon8:c.C916T:p.R306X,TP53:NM_001126114:exon8:c.C916T:p.R306 } \\
\text { X,TP53:NM_001276695:exon8:c.C799T:p.R267X,TP53:NM_001276696:exon8:c.C799 } \\
\text { T:p.R267X,TP53:NM_001276760:exon8:C.C799T:p.R267X,TP53:NM_001276761:exon } \\
\text { 8:c.C799T:p.R267X }\end{array}$ \\
\hline chr18 & 42456670 & 42456670 & - & TCTT & rs3085861 & exonic & SETBP1 & frameshift insertion & SETBP1:NM_001130110:exon4:c.681_682insTCTT:p.T228Sfs*8 \\
\hline
\end{tabular}

Supplementary Table 2: List of curated mutations identified in NEC1452 PDX tumors by exome sequencing.

\begin{tabular}{|c|c|c|c|c|c|c|c|c|c|}
\hline $\mathrm{Chr}$ & Start & End & Ref & Alt & avsnp150 & Func & Gene & ExonicFunc & AAChange \\
\hline chr1 & 89835209 & 89835209 & & A & rs75966734 & exonic & GBP6 & nonsynonymous SNV & GBP6:NM_198460:exon3:C.G295A:p.E99K \\
\hline chr2 & 183066203 & $\mid 183066203$ & c & $\mathrm{T}$ & rs369181374 & exonic & PDE1A & nonsynonymous SNV & 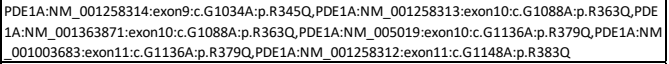 \\
\hline chr4 & 1019055 & 1019056 & CA & - & rs145808953 & exonic & FGFRL1 & frameshift deletion & $\begin{array}{l}\text { FGFRL1:NM_021923:exon6:c.1435_1436del:p.H485LLf*66,FGFRL1:NM_001004356:exon7:c.1435_1436de } \\
\text { l:p.p4485Lffs*66,FGFRL1:NM_001004358:exon7:c.1435_1436del:p.H485Lff*66,FGFRL1:NM_001370296:ex } \\
\text { on7:c.1435_1436del:p.H485Lff*66 }\end{array}$ \\
\hline chr4 4 & 72897699 & 728976996 & & & rs5699440022 & exonic & NPFFR2 & frameshift deletion & NPFFR2:NM_004885:exon1:c.81delG:p.R29Gfs*35 \\
\hline chr5 & 112116592 & 112116592 & c & $\mathrm{T}$ & rs587781392 & exonic & APC & stopgain & 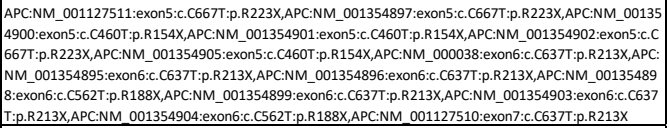 \\
\hline chr5 & \begin{tabular}{|l|l|}
112151184 \\
\end{tabular} & 112151184 & & $G$ & rs1064793022 & intronic & APC & nan & nan \\
\hline chr5 & 180043973 & 180043973 & & A & rs149033942 & exonic & FLT4 & nonsynonymous SNV & $\begin{array}{l}\text { FLLT4:NM_001354989:exon22:C.C3023T:p.P1008L,FLT4:NM_002020:exon22:c.C3023T:p.P1008L,FLT4:NM_ } \\
\text { 182925:exon22:c.C3023T:p.P1008L }\end{array}$ \\
\hline chr6 & 80717709 & 80717709 C & & c & nan & exonic & TTK & nonsynonymous SNV & TTK:NM_001166691:exon3:c.G323C:p.S108T,TTK:NM_003318:exon3:c.G323C:p.S108T \\
\hline chr6 & 80717710 & $80717710 \mathrm{~T}$ & & A & nan & exonic & TTK & nonsynonymous SNV & TTK:NM_001166691:exon3:c.T324A:p.S108R,TTK:NM_003318:exon3:c.T324A:p.S108R \\
\hline chr6 & 154567863 & 154567863 & C & $\mathrm{T}$ & rs34427887 & exonic & IPCEF1;OP & Nstopgain & OPRM1:NM_001008503:exon4:c.C1201T:p.R401X \\
\hline chr7 & 97736519 & $97736521 \mathrm{C}$ & GCT & - & rs776837018 & exonic & LMTK2 & nonframeshift deletion & LMTK2:NM_014916:exon1:c.30_32del:p.L16del \\
\hline chr9 & 390478 & $390478 \mid A$ & A & G & nan & exonic & DOCK8 & nonsynonymous SNV & $\begin{array}{l}\text { DOCK8:NM_001190458:exon22:.A2582G:p.H861R,DOCK8:NM_001193536:exon23:C.A2678G:p.H893R,DO } \\
\text { CK8:NM_203447:exon24:C.A2882G:p.H961R }\end{array}$ \\
\hline chr9 & $139235526 \mid$ & 139235526 & & A & rs59873903 & exonic & GPSM1 & stopgain & GPSM1:NM_015597:exon9:c.C1283A:p.S428X \\
\hline chr10 & \begin{tabular}{|c|c|}
103530307 \\
\end{tabular} & 103530307 & & A & nan & exonic & FGF8 & nonsynonymous SNV & $\begin{array}{l}\text { FGF8:NM_001206389:exon5:C.A202T:p.N688Y,FGF8:NM_006119:exon5:C.A427T:p.N143Y,FGF8:NM_0331 } \\
\text { 65:exon5:C.A394T:p.N132Y,FGF8:NM_033163:exon6:CA514T:p.N172Y,FGF8:NM_033164:exon6:CA481T: } \\
\text { p.N161Y }\end{array}$ \\
\hline chr13 & 48951086 & 48951086 & & 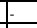 & nan & exonic & RB1 & frameshift deletion & RB1:NM_000321:exon13:c.1248delG:p.R418Efs*2 \\
\hline chr17 & 7578212 & 7578212 & G & A & rs397516436 & exonic & TP53 & stopgain & 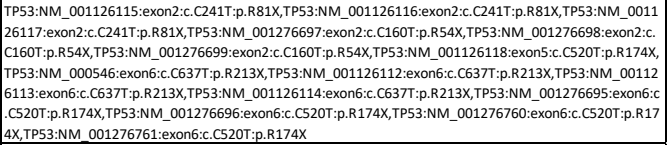 \\
\hline chr19 & 18499449 & $\left.18499449\right|^{T}$ & & G & rs372120002 & exonic & GDF15 & nonsynonymous SNV & GDF15:NM_004864:exon2:c.T631G:p.C211G \\
\hline
\end{tabular}


Supplementary Table 3: Short Tandem Repeats analyses of NEC913 PDX tumors and patient periphery blood mononuclear cells.

\begin{tabular}{|c|c|c|c|c|}
\hline \multirow[b]{2}{*}{ Marker Name } & \multicolumn{2}{|c|}{1} & \multicolumn{2}{|c|}{2} \\
\hline & $\begin{array}{l}\text { Sample } \\
\text { Results }\end{array}$ & 913 blood & $\begin{array}{l}\text { Sample } \\
\text { Results }\end{array}$ & $\begin{array}{c}913 \text { Blood } \\
\text { (IBA\# 11049- } \\
20-01)\end{array}$ \\
\hline AMEL & $\mathrm{X}, \mathrm{Y}$ & NA & $\mathrm{x}$ & $X, Y$ \\
\hline CSF1PO & 10,12 & NA & 10 & 10,12 \\
\hline D13S317 & 12 & NA & 12 & 12 \\
\hline D16S539 & 10,11 & NA & 10 & 10,11 \\
\hline D5S818 & 10,12 & NA & 12 & 10,12 \\
\hline D7S820 & 8,11 & NA & 8,11 & 8,11 \\
\hline TH01 & $7,9.3$ & NA & $7,9.3$ & $7,9.3$ \\
\hline TPOX & 8 & NA & 8 & 8 \\
\hline VWA & 16,18 & NA & 16,18 & 16,18 \\
\hline Identity Match & \multicolumn{2}{|c|}{$\mathrm{N} / \mathrm{A}$, see comments } & \multicolumn{2}{|c|}{$>80 \%$, see comments } \\
\hline
\end{tabular}

Supplementary Table 4: Short Tandem Repeats analyses of NEC1452 PDX tumors.

\begin{tabular}{|l|c|c|}
\hline \multirow{2}{*}{ Marker Name } & \multicolumn{2}{|c|}{1} \\
\cline { 2 - 3 } & $\begin{array}{l}\text { Sample } \\
\text { Results }\end{array}$ & 1452 PDX \\
\hline AMEL & $X$ & NA \\
\hline CSF1PO & 10,11 & NA \\
\hline D135317 & 12 & NA \\
\hline D165539 & 12 & NA \\
\hline D5S818 & 10,11 & NA \\
\hline D7S820 & 9,10 & NA \\
\hline TH01 & $9,9.3$ & NA \\
\hline TPOX & 8,10 & NA \\
\hline WWA & 18 & NA \\
\hline Identity Match & N/A, see comments \\
\hline
\end{tabular}




\section{Funding:}

This work was funded by the PDX Center U54CA224083 Supplement Grant awarded to P.H.E., R.C.F., M.S.O., R.G., and by the NET SPORE P50CA174521 to awarded to M.S.O., T.O., A.M.B., and J.R.H. CGT was supported by T32CA148062. The PerkinElmer Lumina S5 was purchased with the NIH S5 1S100D026835-01 grant.

\section{Acknowledgments}

We thank Dr. Mark B. Evers and Courtney M. Townsend for providing us with the BON cell line.

\section{Author Contributions:}

Conceptualization, P.H.E., J.R.H., R.C.F., C.H.F., M.S.O., T.O., A.A., R.G.; Methodology, C.G.T., L.C.B., J.L.M., E.A., S.A., S.C.G., S.H.V., G.L., C.H.C., G.V.B., M.D., R.L., S.A.W.; Software, P.F.C., S.A., S.A.W., T.J.W.; Formal Analysis, P.H.E., A.M.B., J.R.H., R.C.F., S.A, P.F.C.; Resources, R.C.F, A.M.B, A.A.; Data Curation, P.H.E.; Funding acquisition, P.H.E., R.C.F., M.S.O., R.G., J.R.H., A.M.B., T.O.; Original Draft Preparation, P.H.E.; Writing - Review \& Editing, P.H.E., J.R.H., C.G.T., L.C.B., C.H.C., T.J.W., S.A.W., S.A., A.A.; Supervision, P.H.E.; Project Administration, P.H.E., J.R.H., R.C.F. J.L.M.; All authors have read and agreed to the published version of the manuscript.

\section{Institutional Review Board Statement:}

Patient tumor collection was approved by the Institutional Review Board (protocol \# 201708051; Washington University PDX center). All animal experiments have been approved by the Institutional Animal Care and Use Committee (protocol \# 905177; University of lowa).

\section{Informed Consent Statement:}


Non-applicable.

Data Availability Statement:

Please contact the corresponding authors for any additional data.

Conflicts of Interest:

Authors declare no conflict of interest. 


\section{References}

1. Dasari A, Shen C, Halperin D, et al. Trends in the Incidence, Prevalence, and Survival Outcomes in Patients With Neuroendocrine Tumors in the United States. JAMA Oncol. 2017;3(10):1335-1342.

2. Panzuto F, Boninsegna L, Fazio N, et al. Metastatic and locally advanced pancreatic endocrine carcinomas: analysis of factors associated with disease progression. $J$ Clin Oncol. 2011;29(17):2372-2377.

3. Pavel M, O'Toole D, Costa F, et al. ENETS Consensus Guidelines Update for the Management of Distant Metastatic Disease of Intestinal, Pancreatic, Bronchial Neuroendocrine Neoplasms (NEN) and NEN of Unknown Primary Site.

Neuroendocrinology. 2016;103(2):172-185.

4. Dasari A, Mehta K, Byers LA, Sorbye H, Yao JC. Comparative study of lung and extrapulmonary poorly differentiated neuroendocrine carcinomas: A SEER database analysis of 162,983 cases. Cancer. 2018;124(4):807-815.

5. Nagtegaal ID, Odze RD, Klimstra D, et al. The 2019 WHO classification of tumours of the digestive system. Histopathology. 2020;76(2):182-188.

6. AJCC cancer staging manual. Eighth edition. ed. Switzerland: Springer; 2017.

7. Nagtegaal ID, Odze RD, Klimstra D, et al. The 2019 WHO classification of tumours of the digestive system. Histopathology. 2020;76(2):182-188.

8. Venizelos A, Elvebakken H, Perren A, et al. The molecular characteristics of high-grade gastroenteropancreatic neuroendocrine neoplasms. Endocr Relat Cancer. 2021;29(1):114.

9. Yachida S, Totoki Y, Noe M, et al. Comprehensive Genomic Profiling of Neuroendocrine Carcinomas of the Gastrointestinal System. Cancer Discov. 2021.

10. Korse CM, Taal BG, van Velthuysen ML, Visser O. Incidence and survival of neuroendocrine tumours in the Netherlands according to histological grade: experience of two decades of cancer registry. Eur J Cancer. 2013;49(8):1975-1983.

11. Dasari A, Shen C, Halperin D, et al. Trends in the Incidence, Prevalence, and Survival Outcomes in Patients With Neuroendocrine Tumors in the United States. JAMA oncology. 2017;3(10):1335-1342.

12. Shah $\mathrm{MH}$, Goldner WS, Benson AB, et al. Neuroendocrine and Adrenal Tumors, Version 2.2021, NCCN Clinical Practice Guidelines in Oncology. J Natl Compr Canc Netw. 2021;19(7):839-868.

13. Sorbye H, Strosberg J, Baudin E, Klimstra DS, Yao JC. Gastroenteropancreatic highgrade neuroendocrine carcinoma. Cancer. 2014;120(18):2814-2823.

14. Walenkamp AM, Sonke GS, Sleijfer DT. Clinical and therapeutic aspects of extrapulmonary small cell carcinoma. Cancer treatment reviews. 2009;35(3):228-236.

15. Brennan SM, Gregory DL, Stillie A, Herschtal A, Mac Manus M, Ball DL. Should extrapulmonary small cell cancer be managed like small cell lung cancer? Cancer. 2010;116(4):888-895.

16. Conte B, George B, Overman M, et al. High-Grade Neuroendocrine Colorectal Carcinomas: A Retrospective Study of 100 Patients. Clin Colorectal Cancer. 2016;15(2):e1-7.

17. Terashima T, Morizane C, Hiraoka N, et al. Comparison of chemotherapeutic treatment outcomes of advanced extrapulmonary neuroendocrine carcinomas and advanced small-cell lung carcinoma. Neuroendocrinology. 2012;96(4):324-332.

18. Sorbye $\mathrm{H}$, Welin S, Langer SW, et al. Predictive and prognostic factors for treatment and survival in 305 patients with advanced gastrointestinal neuroendocrine carcinoma (WHO G3): the NORDIC NEC study. Annals of oncology : official journal of the European Society for Medical Oncology. 2013;24(1):152-160. 
19. Strosberg JR, Cheema A, Kvols LK. A review of systemic and liver-directed therapies for metastatic neuroendocrine tumors of the gastroenteropancreatic tract. Cancer Control. 2011;18(2):127-137.

20. Ear PH, Li G, Wu M, Abusada E, Bellizzi AM, Howe JR. Establishment and Characterization of Small Bowel Neuroendocrine Tumor Spheroids. J Vis Exp. 2019(152).

21. Tillotson LG, Lodestro C, Hocker M, Wiedenmann B, Newcomer CE, Reid LM. Isolation, maintenance, and characterization of human pancreatic islet tumor cells expressing vasoactive intestinal peptide. Pancreas. 2001;22(1):91-98.

22. Fujii M, Shimokawa M, Date S, et al. A Colorectal Tumor Organoid Library Demonstrates Progressive Loss of Niche Factor Requirements during Tumorigenesis. Cell Stem Cell. 2016;18(6):827-838.

23. Evers BM, Townsend CM, Jr., Upp JR, et al. Establishment and characterization of a human carcinoid in nude mice and effect of various agents on tumor growth. Gastroenterology. 1991;101(2):303-311.

24. Iguchi $\mathrm{H}$, Hayashi I, Kono A. A somatostatin-secreting cell line established from a human pancreatic islet cell carcinoma (somatostatinoma): release experiment and immunohistochemical study. Cancer Res. 1990;50(12):3691-3693.

25. Benten D, Behrang Y, Unrau L, et al. Establishment of the First Well-differentiated Human Pancreatic Neuroendocrine Tumor Model. Mol Cancer Res. 2018;16(3):496-507.

26. Hofving $T$, Arvidsson $Y$, Almobarak B, et al. The neuroendocrine phenotype, genomic profile and therapeutic sensitivity of GEPNET cell lines. Endocr Relat Cancer. 2018;25(3):367-380.

27. Parekh D, Ishizuka J, Townsend CM, Jr., et al. Characterization of a human pancreatic carcinoid in vitro: morphology, amine and peptide storage, and secretion. Pancreas. 1994;9(1):83-90.

28. Pfragner R, Behmel A, Hoger $\mathrm{H}$, et al. Establishment and characterization of three novel cell lines - P-STS, L-STS, H-STS - derived from a human metastatic midgut carcinoid. Anticancer Res. 2009;29(6):1951-1961.

29. Hernandez Vargas S, Kossatz S, Voss J, et al. Specific Targeting of Somatostatin Receptor Subtype-2 for Fluorescence-Guided Surgery. Clinical cancer research : an official journal of the American Association for Cancer Research. 2019;25(14):43324342.

30. Hernandez Vargas S, Lin C, Voss J, et al. Development of a drug-device combination for fluorescence-guided surgery in neuroendocrine tumors. J Biomed Opt. 2020;25(12).

31. Grozinsky-Glasberg S, Shimon I, Rubinfeld $\mathrm{H}$. The role of cell lines in the study of neuroendocrine tumors. Neuroendocrinology. 2012;96(3):173-187.

32. Kolby L, Bernhardt $\mathrm{P}$, Ahlman $\mathrm{H}$, et al. A transplantable human carcinoid as model for somatostatin receptor-mediated and amine transporter-mediated radionuclide uptake. Am J Pathol. 2001;158(2):745-755.

33. Chamberlain CE, German MS, Yang K, et al. A Patient-derived Xenograft Model of Pancreatic Neuroendocrine Tumors Identifies Sapanisertib as a Possible New Treatment for Everolimus-resistant Tumors. Mol Cancer Ther. 2018;17(12):2702-2709.

34. Yachida S, Vakiani E, White CM, et al. Small cell and large cell neuroendocrine carcinomas of the pancreas are genetically similar and distinct from well-differentiated pancreatic neuroendocrine tumors. Am J Surg Pathol. 2012;36(2):173-184.

35. Kawasaki K, Toshimitsu K, Matano M, et al. An Organoid Biobank of Neuroendocrine Neoplasms Enables Genotype-Phenotype Mapping. Cell. 2020.

36. Pettengill OS, Sorenson GD, Wurster-Hill DH, et al. Isolation and growth characteristics of continuous cell lines from small-cell carcinoma of the lung. Cancer. 1980;45(5):906918. 
37. Baillie-Johnson H, Twentyman PR, Fox NE, et al. Establishment and characterisation of cell lines from patients with lung cancer (predominantly small cell carcinoma). $\mathrm{Br} \mathrm{J}$ Cancer. 1985;52(4):495-504.

38. Gock M, Mullins CS, Harnack C, et al. Establishment, functional and genetic characterization of a colon derived large cell neuroendocrine carcinoma cell line. World $\mathrm{J}$ Gastroenterol. 2018;24(33):3749-3759.

39. Detjen K, Hammerich L, Ozdirik B, et al. Models of Gastroenteropancreatic Neuroendocrine Neoplasms: Current Status and Future Directions. Neuroendocrinology. 2021;111(3):217-236.

40. Krieg A, Mersch S, Boeck I, et al. New model for gastroenteropancreatic large-cell neuroendocrine carcinoma: establishment of two clinically relevant cell lines. PLoS One. 2014;9(2):e88713.

41. Li M, Sagastume EA, Lee D, et al. (203/212)Pb Theranostic Radiopharmaceuticals for Image-guided Radionuclide Therapy for Cancer. Curr Med Chem. 2020;27(41):70037031.

42. Kaemmerer D, Reimann C, Specht E, et al. Differential expression and prognostic value of the chemokine receptor CXCR4 in bronchopulmonary neuroendocrine neoplasms. Oncotarget. 2015;6(5):3346-3358.

43. Werner RA, Kircher S, Higuchi T, et al. CXCR4-Directed Imaging in Solid Tumors. Front Oncol. 2019;9:770. 